\title{
A REFORMULATION OF THE RADON-NIKODYM THEOREM
}

\section{JONATHAN LEWIN AND MIRIT LEWIN}

ABSTRACT. The Radon-Nikodym theorems of Segal and Zaanen are principally concerned with the classification of those measures $\mu$ for which any $\lambda \ll \mu$ is given in the form

$$
\lambda(A)=\int_{A} g d \mu
$$

for all sets $A$ of finite $\mu$ measure.

This paper is concerned with the characterization of those pairs $\lambda, \mu$ for which the equality (i) holds for every measurable set $A$, and introduces a notion of compatibility that essentially solves this problem. In addition, some applications are made to Radon-Nikodym theorems for regular Borel measures.

1. Introduction. The sharpest known form of the Radon-Nikodym theorem to date is due to Segal and appears in Segal [6] and Zaanen [7]. The purpose of this paper is to answer some basic questions left unasked by Segal and Zaanen and, at the same time, attempt to throw some light upon the Radon-Nikodym theorems for regular Borel measures, and upon the way some of these can be made to follow simply from their abstract counterparts.

Notation. Measures $\mu$ and $\lambda$ will be nonnegative and defined on a sigma algebra, $\Sigma$, of subsets of a set $S$. By a measurable set we shall simply mean a member of $\Sigma$. A measurable set $A$ is called $\mu$-null if $\mu(A)=0$. If every measurable subset of $A$ with finite $\mu$ measure is $\mu$-null, then we say that $A$ is $\mu$-locally null. The nonnull locally null sets of a measure $\mu$ can be "killed" by replacing $\mu$ by its corresponding contracted measure $\mu^{*}$ defined by $\mu^{*}(A)=\sup \{\mu(B): B \subset A$ and $\mu(B)<\infty\}$. (See Zaanen $[7, \$ 10]$.) Clearly, $\mu=\mu^{*}$ iff $\mu$ has no nonnull locally null sets. If every $\mu$-null set is $\lambda$-null, then we say that $\lambda$ is absolutely continuous with respect to $\mu$ and write $\lambda \ll \mu$. The relation $d \lambda=g d \mu$ is said to hold

Received by the editors August 24, 1973 and, in revised form, December 18, 1973.

AMS (MOS) subject classifications (1970). Primary 28A15, 28A30.

Key words and phrases. Radon-Nikodym theorem, measure space, Borel measure, absolute continuity, differentiation of measures. 
$\mu$-locally (respectively, $\lambda$-locally) in a set $A$ if we have

$$
\lambda(B)=\int_{B} g d \mu
$$

for every $\mu$-sigma finite ( $\lambda$-sigma finite) subset $B$ of $A$. If ( 1 ) holds for every measurable subset $B$ of $A$, then the relation $d \lambda=g d \mu$ is said to hold globally in $A$. If $A$ is not mentioned then it will be understood that $A=S$. The minimum of measures $\mu$ and $\lambda$ (in the lattice of nonnegative measures defined on $\Sigma$ ) will be denoted as usual by $\mu \wedge \lambda$. A cross section of a measure $\mu$ is a family $\left\{g_{A}: \mu(A)\right.$ is sigma finite $\}$ where for each $A$, $g_{A}$ is a nonnegative measurable function which vanishes $\mu$-almost everywhere outside $A$, and such that whenever $A$ and $B$ are $\mu$-sigma finite sets, the functions $g_{A}$ and $g_{B}$ agree $\mu$-almost everywhere in $A \cap B$.

Theorem 1.1 (The classical Radon-Nikodym theorem). If $\lambda .<\mu$ and $\mu$ is sigma finite, then there exists a measurable function $g$ such that $d \lambda=$ $g d \mu$ globally.

Note that $\lambda$ need not be sigma finite (Halmos [1, p. 131]). From this theorem the following general result follows easily (Zaanen [7, Theorem 7.3]).

Theorem 1.2. If $\lambda \ll \mu$, then there exists a cross section $\left\{g_{A}\right\}$ of $\mu$ such that whenever a set $A$ is $\mu$-sigma finite, we have $d \lambda=g_{A} d \mu$ globally in $A$.

The work of Segal and Zaanen is largely concerned with the question as to when every cross section $\left\{g_{A}\right\}$ of a given measure $\mu$ can be pieced together into a single measurable function $g$ which will agree $\mu$-almost everywhere with each function $g_{A}$ on the corresponding set $A$. They show that this property of $\mu$ (called the $R \cdot N$ property) is equivalent to the condition that given any $\lambda$ satisfying $\lambda \ll \mu$, there exists a measurable function $g$ such that $d \lambda=g d \mu \mu$-locally. Among the other equivalent conditions that they give are Segal's well-known criterion of localizability and the condition that $L^{1}(\mu)^{*}=L^{\infty}(\mu)$. In addition, they show that if $\mu$ is decomposable into finite measures (Hewitt and Stromberg [3, $\$ 19.25$ and 19.27], and Zaanen [7, Theorem 7.1]), then $\mu$ has the $R \cdot N$ property. Of course, every sigma finite measure has the $R \cdot N$ property.

However, Segal and Zaanen do not give conditions under which the relation $d \lambda=g d \mu$ will be valid globally, and it is with this question that we are concerned here. 
2. Compatibility. If $\lambda$ has no nonnull locally null sets, then whenever $\lambda(A)=\infty, A$ must contain subsets of arbitrarily large finite $\lambda$ measure, and consequently, if for a given $\mu$, the relation $d \lambda=g d \mu$ holds $\lambda$-locally, it actually holds globally. However, even if neither $\mu$ nor $\lambda$ has nonnull locally null sets, the relation $d \lambda=g d \mu$ can quite easily hold $\mu$-locally without holding globally. We may look, for instance, at the famous "Saks example" in which $\lambda$ is Lebesgue measure and $\mu$ is counting measure on the measurable subsets of $[0,1]$. In this example we see that $d \lambda=0 d \mu$ $\mu$-locally, but that there is no function $g$ such that $d \lambda=g d \mu$ globally.

Definition. We say that $\lambda$ is compatible with $\mu$ if whenever $0<\lambda(A)$ $<\infty$, there exists a measurable subset $B$ of $A$ such that $\lambda(B)>0$ and $\mu(B)<\infty$.

Note that any measure is compatible with a sigma finite measure. The following theorem is clear:

Theorem 2.1. A necessary condition for the existence of a measurable function $g$ such that $d \lambda=g d \mu$ globally is that $\lambda \ll \mu$ and that $\lambda$ be com. patible with $\mu$.

The converse of Theorem 2.1 does not hold, as we see in the following three examples:

Example 1. (Cf. Zaanen [7, Example 4] or Royden [4, p. 249, Problem 46].) Let $M$ and $N$ be uncountable sets such that $|M|<|N|$, and let $S=$ $M \times N$. Call a set $A$ measurable if, for every horizontal or vertical line $L$ in $M \times N$, either $L \cap A$ or $L \backslash A$ is countable. For each measurable $A$, let $\mu(A)$ be the number of horizontal or vertical lines $L$ such that $L \backslash A$ is countable, and define $\lambda(A)$ similarly, counting the horizontal lines only. Then although neither $\mu$ nor $\lambda$ has nonnull locally null sets and $\lambda$ is compatible with $\mu$ and $\lambda \ll \mu$, there is no function $g$ such that $d \lambda=g d \mu$ globally, nor even a $g$ such that $d \lambda=g d \mu \mu$-locally. Of course, $\mu$ does not have the $R \cdot N$ property.

Example 2. Let $\mathbf{R}_{d}$ be the discrete group of reals and let $\lambda$ be Haar measure on the locally compact group $\mathbf{R}_{d} \times \mathbf{R}$. Define $\mu$ to be $\lambda$ plus the counting measure on $\mathbf{R}_{d} \times\{0\}$. Then $\lambda$ is compatible with $\mu$ and $\lambda \ll \mu$, and although it is easy to find $g$ such that $d \lambda=g d \mu \mu$-locally, there is no $g$ such that $d \lambda=g d \mu$ globally.

Example 3. Let $\mu$ be Haar measure on $\mathbf{R}_{d} \times \mathbf{R}$ and let $\lambda=\mu^{*}$. Then $\lambda$ is compatible with $\mu, \lambda \ll \mu, \lambda$ has no nonnull locally null sets, and $d \lambda=1 d \mu \mu$-locally, but there is no $g$ such that $d \lambda=g d \mu$ globally. 
If $\lambda$ is finite, the pathology of the above examples disappears.

Theorem 2.2. If $\lambda(S)<\infty, \lambda \ll \mu$, and $\lambda$ is compatible with $\mu$, then there exists a measurable function $g$ such that $d \lambda=g d \mu$ globally.

Proof. Define $\alpha=\sup \{\lambda(A): \mu(A)$ is sigma finite $\}$, and choose an increasing sequence $\left\{A_{n}\right\}$ of measurable sets such that $\lambda\left(A_{n}\right) \rightarrow \alpha$ as $n \rightarrow \infty$, and define $A=\bigcup_{n=1}^{\infty} A_{n}$. Then $\lambda(A)=\alpha$ and $A$ is $\mu$-sigma finite. Clearly $\lambda(S \backslash A)=0$, for otherwise there would exist a measurable subset $B$ of $S \backslash A$ such that $\lambda(B) \neq 0$ and $\mu(B)<\infty$, and we would have $\lambda(A \cup B)>\alpha$, in spite of the fact that $\mu(A \cup B)$ is sigma finite. Using the classical Radon-Nikodym theorem, choose a measurable function $g$ such that $d \lambda=g d \mu$ holds globally inside $A$, and define $g(x)=0$ for every $x$ outside $A$. Clearly, $d \lambda=$ $g d \mu$ globally. This completes the proof.

In the Saks example (above), $\lambda$ fails to be compatible with $\mu$ in a rather strong way. In fact, $\lambda$ is totally incompatible with $\mu$, by which we mean that $\lambda(A) \neq 0 \Longrightarrow \mu(A)=\infty$. By returning to one of the classical proofs of the Radon-Nikodym theorem, we can improve Theorem 2.2.

Theorem 2.3. Suppose $\lambda$ is finite. Then $\lambda$ can be uniquely decomposed into the sum of three measures $\lambda_{s}, \lambda_{t}$ and $\lambda_{c}$, where $\lambda_{s}$ is singular to $\mu$, $\lambda_{t}$ is totally incompatible with $\mu$, and $\lambda_{c}$ is both absolutely continuous and compatible with $\mu$. A fortiori there exists $g \in L^{1}(\mu)$ such that $\lambda(A)=$ $\lambda_{s}(A)+\lambda_{t}(A)+\int_{A} g d \mu$, for every measurable set $A$.

Proof. Define $\phi=\lambda+\mu$, and let $T$ be the functional on $L^{2}(\phi)$ defined by $T(f)=\int_{S} f d \lambda$ for every $f \in L^{2}(\phi)$. Then since

$$
|T(f)|^{2} \leq\left|\int_{S} f d \lambda\right|^{2} \leq \lambda(S) \int_{S}|f|^{2} d \lambda \leq \lambda(S) \int_{S}|f|^{2} d \phi
$$

we see that $\|T\| \leq \sqrt{\lambda(S)}$. Therefore, there exists $h \in L^{2}(\phi)$ such that $0 \leq$ $h \leq 1$ and $\int_{S} f d \lambda=\int_{S} f h d \phi$ for every $f \in L^{2}(\phi)$. The proof is now concluded by defining $\lambda_{s}, \lambda_{t}$ and $\lambda_{c}$ to be the respective restrictions of $\lambda$ to the sets of points $x$ where $h(x)$ is, respectively, one, zero, or satisfies $0<h(x)<$ 1 , and defining $g=(h /(1=h))_{C}$, where $C=\{x: 0<h(x)<1\}$.

It follows from these theorems that even if the measure $\mu$ fails to have the $R-N$ property, we can nevertheless obtain the relation $d \lambda=g d \mu$ globally when $\lambda$ is finite, $\lambda \ll \mu$ and $\lambda$ is compatible with $\mu_{0}$. Furthermore we obtain

Corollary 2.4. Let $T$ be a bounded linear functional on $L^{1}(\mu)$, and suppose there exists a constant $K$ such that $|T(f)| \leq K\|f\|_{\infty}$ for every 
$f \in L^{1}(\mu)$. Then there exists $g \in L^{\infty}(\mu)$ such that $T(f)=\int f g d \mu$ for every $f \in L^{1}(\mu)$.

Proof. Note that by $\|f\|_{\infty}$ we mean the least number $\alpha$ such that $\{x: f(x)>\alpha\}$ is $\mu$-locally null. We may assume $T \geq 0$. Now for every measurable set $A$ define $\lambda(A)=\sup \left\{T\left(\chi_{B}\right): B \subseteq A\right.$ and $\left.\mu(B)<\infty\right\}$. Then the measure $\lambda$ is finite, $\lambda \ll \mu$ and $\lambda$ is compatible with $\mu$, and so by Theorem 2.2 there exists $g$ such that $d \lambda=g d \mu$ globally, and it is easy to see that the function $g$ has all the required properties.

3. The case $\lambda$ infinite. We have remarked that if $\lambda$ is infinite, then Theorem 2.1 has no general converse. Positive results may be obtained, however, by imposing certain other restrictions on $\mu$ and $\lambda$.

Theorem 3.1. Suppose neither $\lambda$ nor $\mu$ has nonnull locally null sets, $\lambda \ll \mu, \lambda$ is compatible with $\mu$, and that there exists a measurable function $g$ such that $d \lambda=g d \mu \mu$-locally. (The latter condition would hold, for example, if $\mu$ had the $R \cdot N$ property.) Then for any such function $g$ we have $d \lambda=g d \mu$ globally.

Proof. We need only show that $d \lambda=g d \mu \lambda$-locally. Suppose $\lambda(A)<\infty$ and, using Theorem 2.2, choose a function $h$ defined in $A$ such that $d \lambda=$ $h d \mu$ globally in $A$. Clearly $h$ and $g$ agree $\mu$-locally almost everywhere, and therefore $\mu$-almost everywhere, in $A$. Hence $\lambda(A)=\int_{A} h d \mu=\int_{A} g d \mu$, and the result follows.

Theorem 3.2. Suppose neither $\lambda$ nor $\mu$ has nonnull locally null sets, $\lambda \ll \mu, \lambda$ is compatible with $\mu$, and that $\lambda$ has the $R-N$ property. Then there exists a measurable function $g$ such that given any measurable set $A$, there exists a measurable subset $B$ of $A$ such that $\lambda(A)=\lambda(B)=\int_{B} g d \mu$.

Proof. For every set $A$ of sigma finite $\lambda$ measure, choose a measurable function $g_{A}$ which vanishes outside $A$ and satisfies $d \lambda=g_{A} d \mu$ globally in $A$ (see Theorem 2.2). Note that if $A$ and $B$ are two sets of sigma finite $\lambda$ measure, then $g_{A}$ and $g_{B}$ clearly agree $\mu$-locally almost everywhere, and therefore $\mu$-almost everywhere, in $A \cap B$. Therefore since $\lambda \ll \mu$ we see that $g_{A}$ and $g_{B}$ agree $\lambda$-almost everywhere in $A \cap B$, and it follows that $\left\{g_{A}: \lambda(A)\right.$ is sigma finite $\}$ is a cross section of $\lambda$, and so there exists a measurable function $g$ such that whenever $\lambda(A)$ is sigma finite, $g$ and $g_{A}$ agree $\lambda$-almost everywhere in $A$. 
Now suppose that $\lambda(A)<\infty$, and define $B=\left\{x \in A: g_{A}(x)=g(x)\right\}$. Then $\lambda(A)=\lambda(B)=\int_{B} g_{A} d \mu=\int_{B} g d \mu$, and, in particular, $\lambda(A) \leq \int_{A} g d \mu$.

On the other hand, if $\lambda(A)=\infty$, we see that $A$ contains subsets $B$ of arbitrarily large finite $\lambda$ measure, and we obtain

$$
\begin{aligned}
\int_{A} g d \mu & \geq \sup \left\{\int_{B} g d \mu: B \subseteq A \text { and } \lambda(B)<\infty\right\} \\
& \geq \sup \{\lambda(B): B \subseteq A \text { and } \lambda(B)<\infty\}=\infty .
\end{aligned}
$$

This completes the proof.

Roughly speaking, small measures are more likely to have the $R-N$ property than large ones. Therefore the following combination of Theorems 3.1 and 3.2 is of interest.

Theorem 3.3. Suppose neither $\lambda$ nor $\mu$ has nonnull locally null sets, $\lambda \ll \mu, \lambda$ is compatible with $\mu$, and $\mu \wedge \lambda$ has the $R-N$ property. Then there exists a measurable function $g$ such that the conclusions of Theorem 3.2 bold.

Proof. The result follows on combining Theorems 3.1 and 3.2, after noticing that $\lambda<<\mu \wedge \lambda$, and $\mu \wedge \lambda$ clearly has no nonnull locally null sets.

4. Regular Borel measures. By a regular Borel measure on a locally compact space $S$ we mean a measure $\mu$ constructed from a nonnegative functional as in Rudin [5, Theorem 2.14], or Hewitt and Ross [2, Chapter 11], or Hewitt and Stromberg $[3, \S \S 9$ and 10]. $\mu$ is really an outer measure defined on all subsets of $S$, and is a measure on the family $\Sigma_{\mu}$ of $\mu$-measurable sets which contains all Borel sets. Note that if $\lambda$ and $\mu$ are regular Borel measures, and every $\mu$-null set is $\lambda$-locally null (for example, we might have $\lambda \ll \mu$ ), then $\Sigma_{\mu} \subset \Sigma_{\lambda}$, so we may regard both $\lambda$ and $\mu$ as defined on the same sigma algebra $\Sigma_{\mu}$. Furthermore, $\lambda$ is automatically compatible with $\mu$, for if $0<\lambda(A)<\infty$, then there is a compact subset $K$ of $A$ such that $\lambda(K)>0$, and of course $\mu(K)<\infty$. Finally, we note from Hewitt and Stromberg [3, Corollary 19.31] that a regular. Borel measure has the $R-N$ property. 1

From these observations, we obtain immediate proofs of the following three theorems (the first two of which are well known).

${ }^{1}$ In saying that a regular Borel measure has the $R-N$ property, we regard $\mu$ as defined on all $\mu$-measurable sets, not only on the Borel sets. 
Theorem 4.1 (Hewitt and Stromberg [3, Theorem 19.32]). Let $\mu$ be a regular Borel measure, let $\lambda$ be any measure defined on $\Sigma_{\mu}$ and suppose $\lambda \ll \mu$. Then there is a $\mu$-measurable function $g$ such that $d \lambda=g d \mu$ $\mu$-locally.

Proof. The result follows at once since $\mu$ has the $R-N$ property.

Theorem 4.1 is essentially the same result as Hewitt and Ross [2, Theorem 12.17]. (It really is!)

Theorem 4.2 (Hewitt and Ross [2, Theorem 14.19]). Let $\lambda$ and $\mu$ be regular Borel measures, suppose $\lambda .<\mu$ and suppose $\lambda(S)<\infty$. Then there is a $\mu$-measurable function $g$ such that $d \lambda=g d \mu$ globally.

Proof. The result follows at once from Theorem 2.2 since $\lambda$ is compatible with $\mu$.

The following result follows at once from Theorem 3.1.

Theorem 4.3. If $\lambda$ and $\mu$ are regular Borel measures and neither $\lambda$ nor $\mu$ has nonnull locally null sets and $\lambda . \ll \mu$, there is a $\mu$-measurable function $g$ such that $d \lambda=g d \mu$ globally.

Actually, one can do better than this, but the sharper result must be proved directly:

Theorem 4.4. Let $\lambda$ and $\mu$ be regular Borel measures and suppose that every $\mu$-null set is $\lambda$-locally null. Then there is a $\mu$-measurable func. tion $g$ such that $d \lambda=g d \mu \lambda$-locally.

Proof. Choose a family $\mathcal{F}$ of compact sets for the measure $\lambda$ as in Hewitt and Ross [2, Theorem 11.39]. Let $N=S \backslash \bigcup \mathcal{F}$. Then $N$ is $\lambda$-locally null. For each $F \in \mathcal{F}$ the measure $\lambda_{F}$, defined by $\lambda_{F}(A)=\lambda(A \cap F)$ for all $\Sigma_{\mu}$-measurable sets $A$, is a finite measure compatible with $\mu$, and so by Theorem 2.2 there is a $\mu$-measurable function $g_{F}$ such that $d \lambda_{F}=$ $g_{F} d \mu$ globally. We may assume that $g_{F}$ is zero outside $F$. Define $g$ as follows: If $x \in N$ then $g(x)=0$, and if $x \in F \in \mathcal{F}$ then $g(x)=g_{F}(x)$. Then $g$ is $\mu$-measurable: (This may be a little surprising, since there is no reason to expect $N \in \Sigma_{\mu}$.) For if $a<0$ then $\{x: g(x)>\alpha\}=S$, and if $\alpha \geq 0$, then $\{x: g(x)>\alpha\}$ is $\mu$-measurable since its intersection with any compact set $K$ is of the form $\bigcup_{n=1}^{\infty} K \cap\left\{x: g_{F_{n}}(x)>\alpha\right\} \in \Sigma_{\mu^{*}}$ Clearly $d \lambda=g d \mu$ $\lambda$-locally.

This gives the ultimate result for regular Borel measures: 
Corollary 4.5. Let $\lambda$ and $\mu$ be regular Borel measures, suppose $\lambda$ has no nonnull locally null sets and $\lambda<<\mu$. Then there exists a $\mu$-measurable function $g$ such that $d \lambda=g d \mu$ globally.

Remark. Example 2 shows that in Corollary 4.5 we cannot dispense with the assumption that $\lambda$ has no nonnull locally null sets.

Example 3 shows that in Theorem 4.4 and Corollary 4.5 we cannot dispense with the assumption that $\lambda$ be regular. It also shows that in Theorem 3.1 we cannot dispense with the assumption that $\mu$ have no nonnull locally null sets.

Note however, that in Theorem 4.4 and Corollary 4.5, $\mu$ may have nonnull locally null sets.

\section{REFERENCES}

1. P. R. Halmos, Measure theory, Van Nostrand, Princeton, N. J., 1950. MR 11, 504 .

2. E. Hewitt and K. A. Ross, Abstract harmonic analysis. Vol. I: Structure of topological groups. Integration theory, group representations, Die Grundlehren der math. Wissenschaften, Band 115, Academic Press, New York, Springer-Verlag, Berlin, 1963. MR $28 \# 158$.

3. E. Hewitt and K. Stromberg, Real and abstract analysis. A modern treatment of the theory of functions of a real variable, Springer-Verlag, New York, 1965. MR 32 \#5826.

4. H. L. Royden, Real analysis, Macmillan, New York, 1968.

5. Walter Rudin, Real and complex analysis, McGraw-Hill, New York, 1966. MR $35 \# 1420$.

6. I. E. Segal, Equivalences of measure spaces, Amer. J. Math. 73 (1951), 275-313. MR 12, 809.

7. A. C. Zaanen, The Radon-Nitiodym theorem. I, II, Nederl. Akad. Wetensch. Proc. Ser. A 64 = Indag. Math. 23 (1961), 157-187. MR 26 \#3862.

DEPARTMENT OF MATHEMATICS, THE BEN GURION UNIVERSITY OF THE NEGEV, BEER SHEVA, ISRAEL

Current address (both authors): Department of Mathematics, University of the Witwatersrand, Johannesburg, South Africa 